\title{
Cooperative Diversity in the Presence of Impulsive Noise
}

\author{
Suhail Al-Dharrab, Student Member, IEEE, and Murat Uysal, Senior Member, IEEE
}

\begin{abstract}
Although there already exists a rich literature on cooperative diversity, current results are mainly restricted to the conventional assumption of additive white Gaussian noise (AWGN). AWGN model realistically represents the thermal noise at the receiver, but ignores the impulsive nature of atmospheric noise, electromagnetic interference, or man-made noise which might be dominant in many practical applications. In this paper, we investigate the performance of cooperative communication over Rayleigh fading channels in the presence of impulsive noise modeled by Middleton Class A noise. Specifically, we consider a multi-relay network with amplify-and-forward relaying. Through the derivations of pairwise error probability, we quantify the diversity advantages. Based on the minimization of a union bound on the error rate performance, we formulate optimal power allocation schemes and demonstrate significant performance gains over their counterparts with equal power allocation. An extensive Monte Carlo simulation is also presented to illustrate the performance of cooperative schemes in various impulsive environments.
\end{abstract}

Index Terms-Cooperative diversity, impulsive noise, spacetime block codes, pairwise error probability, power allocation.

\section{INTRODUCTION}

A major performance-limiting factor in wireless communication systems is multipath-induced fading. Fading severely degrades the link performance and powerful countermeasures such as diversity techniques should be employed to maintain an acceptable performance. Spatial diversity involves the deployment of multiple transmit and/or receive antennas and is commonly used to improve the link reliability and/or throughput in wireless systems. In various applications where spacing limits the deployment of multiple antennas, cooperative diversity is considered an effective fading-mitigation technique [1]-[5].

Cooperative diversity takes advantage of the broadcast nature of wireless transmission where a transmitted signal can be overheard by many unintended nodes. If these unintended nodes (or relays) are willing to share their resources with the source node, they can together create a virtual antenna array to extract the spatial diversity in a distributed fashion. The concept of cooperative diversity can be traced back to

Manuscript received September 26, 2008; revised March 18, 2009; accepted June 9, 2009. The associate editor coordinating the review of this paper and approving it for publication was Y.-C. Ko.

This paper was presented in part at IEEE WCNC'09, Budapest, Hungary, April 2009. The work of M. Uysal is supported, in part, by an NSERC (Natural Sciences and Engineering Research Council of Canada) Collaborative Research and Development Grant (CRDPJ348999-06).

The authors are with the Department of Electrical and Computer Engineering, University of Waterloo, Waterloo, ON, N2L3G1, Canada (e-mail: \{sialdhar, muysal\}@ece.uwaterloo.ca).

Digital Object Identifier 10.1109/TWC.2009.081290
Van der Meulen's earlier work [6] on relay channels. The recent surge of interest however has followed after the works of Laneman et al. and Sendonaris et al. [1]-[4]. Since then, a large number of publications have appeared in the area of cooperative communications investigating variety of topics such as information theoretic bounds, cooperation protocols, distributed space-time code design, distributed source coding, optimum power allocation, cross-layer design etc. among others. Detailed surveys of various issues in cooperative communication systems can be found in recent books [7], [8].

The current rich literature on cooperative diversity is mainly limited to the conventional assumption of additive white Gaussian noise (AWGN). AWGN model realistically represents the thermal noise at the receiver, but ignores the impulsive nature of atmospheric noise, electromagnetic interference (EMI), or man-made noise which might be dominant in many applications. For instance, automotive ignition noise, power transmission lines, and arc generating circuit components are examples of impulsive noise sources, which are encountered mainly in metropolitan areas [9]. In indoor wireless communication, devices with electromechanical switches such as electrical motors in elevators, refrigerators units, photocopy machines, and printers are considered as impulsive noise sources. Furthermore, microwave ovens, cash register receipt printers, gas-powered engines, and compressor motors produce impulsive noise on frequency bands which coincide with the operating frequencies of current cellular and wireless local area networks [10], [11].

A widely accepted model for impulsive noise is Middleton's model [12], [13] with three distinct classes. Middleton Class A model is valid when the bandwidth of noise is less than that of the receiver front-end and interference waveforms produce negligible transients at the receiver. If the noise bandwidth exceeds receiver front-end bandwidth and interference waveforms result in significant transients, Class B model becomes valid. Class $\mathrm{C}$, on the other hand, is considered as a mixture of Class A and B models [12].

Middleton Class A model has been widely used in analyzing the performance of communication systems under impulsive noise, see e.g., [14]-[19]. In [17] and [18], Spaulding and Middleton have derived optimum coherent and non-coherent detection rules using maximum likelihood detection for fading and non-fading channels in the presence of impulsive noise. In [15], Haring and Vinck have investigated the performance of coded transmission over impulsive noise through the derivations of Chernoff factor and cut-off rate assuming a MAP (maximum a posteriori) detector. In [19], Tepedelenlioglu and Gao have studied the performance of various diversity reception techniques in impulsive noise. In particular, they have 
derived upper bounds on average bit error rate and investigated the performance of maximal ratio combining (MRC), equal gain combining (EGC), selection combining (SC), and postdetection combining (PDC). In [16], they have extended their work to transmit diversity where they have obtained design criteria for space-time codes. They have further investigated the performance of MIMO (multi-input multi-output) systems with different receiver types including GAR (genie-aided receiver), MAP receiver, and MDR (minimum distance receiver).

To the best of our knowledge, no research results have been published on cooperative diversity in the presence of impulsive noise. Aiming to fill this research gap, our paper presents an error rate performance analysis of cooperative diversity over Rayleigh fading channel in impulsive noise. In particular, we consider a multi-relay system with amplify-andforward relaying and assume Protocol II of [1], [5] which realizes receive diversity in a distributed manner. We obtain upper bounds on pairwise error probability (PEP) expression under Middleton Class A noise assuming the deployment of an MDR. Our results demonstrate that the performance of cooperative system highly depends on the impulsive nature of noise and different diversity orders dominate the performance in different ranges of signal-to-noise ratios (SNRs). We further optimize the bit error rate (BER) performance through proper power allocation among cooperating nodes. The optimization yields gains up to $\sim 3 \mathrm{~dB}$ at a target BER of $10^{-6}$ in a highly impulsive (HI) environment for a two-relay cooperative scheme. In near-Gaussian (NG) impulsive environment, performance gains climb up to $5 \mathrm{~dB}$ depending on the relays' locations.

The rest of the paper is organized as follows: In Section II, we describe the system model introducing fading channel, noise model, and cooperative transmission under consideration. In Section III, we derive PEP expressions and obtain bounds on the error rate performance for spatially dependent and independent impulsive noise assuming MDR deployment. In Section IV, we optimize power allocation based on the minimization of union bound on BER. In Section $\mathrm{V}$, we present extensive Monte Carlo simulations for various impulsive environments. Finally, we conclude in Section VI. In the Appendix, we present GAR and MAP detection rules for cooperative scheme under consideration.

Notation: We use bold upper-case and lower-case letters to denote matrices and vectors respectively. $(.)^{*},(.)^{T}$, and $(.)^{H}$ denote conjugate, transpose, and conjugate transpose respectively. ||$.||$ and $|$.$| denote Frobenius norm and absolute$ value. $\lceil$.$\rceil denotes the ceiling function, \operatorname{diag}($.$) denotes$ diagonal matrix, and $Q($.$) stands for the Gaussian Q-function.$ $I_{N}$ denotes an identity matrix of size $N$.

\section{SySTEM MOdeL}

In our work, we consider a cooperative communication system with $M$ relays over Rayleigh fading channel in the presence of impulsive noise. In the following, we introduce fading channel and noise models followed by the transmission model.

\section{A. Fading Channel Model}

We assume an aggregate channel model which takes into account not only the small-scale fading, but also large-scale path loss to reflect the effect of relays' locations. The fading coefficients over source-to-destination $(S \rightarrow D)$, source-to$i^{\text {th }}$ relay $\left(S \rightarrow R_{i}\right)$, and $i^{\text {th }}$ relay-to-destination $\left(R_{i} \rightarrow D\right)$ links are respectively denoted by $h_{S D}, h_{S R_{i}}$, and $h_{R_{i} D}$. They are assumed to be independent and identically distributed (i.i.d) zero-mean complex Gaussian random variables leading to a Rayleigh fading channel model. Path losses in $S \rightarrow D$, $S \rightarrow R_{i}$, and $R_{i} \rightarrow D$ are respectively denoted by $c d_{S D}^{-\alpha}$, $c d_{S R_{i}}^{-\alpha}$, and $c d_{R_{i} D}^{-\alpha}$ where $d_{S D}, d_{S R_{i}}$, and $d_{R_{i} D}$ are the corresponding distances among nodes, $\alpha$ is path loss coefficient, and $c$ is a constant related to propagation environment. We denote the angle formed by $S \rightarrow R_{i}$ and $R_{i} \rightarrow D$ links by $\phi_{i}$. Normalizing the path losses with respect to the direct path, the relative geometrical gains ${ }^{1}$ are defined as $g_{S R_{i}}=\left(d_{S R_{i}} / d_{S D}\right)^{-\alpha}$ and $g_{R_{i} D}=\left(d_{R_{i} D} / d_{S D}\right)^{-\alpha}[20]$. Path loss ratio can be further defined as

$$
\Theta_{i}=\frac{g_{S R_{i}}}{g_{R_{i} D}}=\left(\frac{d_{R_{i} D}}{d_{S R_{i}}}\right)^{\alpha}
$$

which indicates the relative location of $i^{\text {th }}$ relay with respect to source and destination.

\section{B. Noise Model}

We assume Middleton Class A model to represent the impulsive noise under consideration. Noise at the receiver is given by

$$
n_{r}=n_{g}+n_{i}
$$

where $n_{g}$ represents the thermal noise and is modeled by zero-mean complex-valued Gaussian with variance $\sigma_{g}^{2}$. In (2), $n_{i}$ is the impulsive component and results from the occurrence of interfering waveforms from active interfering sources. Under the assumption that the number of such sources is large enough and they emit independently, occurrence of interferences follow Poisson distribution [21]. Therefore, the probability of having $k$ active interferences (or impulses) is denoted by $\alpha_{k}$ and given by

$$
\alpha_{k}=\exp (-A) \frac{A^{k}}{k !}
$$

where $A$ is called the impulsive index and indicates the average number of impulses during interference time. Probability density function (pdf) of the complex-valued noise at receiver is given by

$$
p\left(n_{r}\right)=\sum_{k=0}^{\infty} \frac{\alpha_{k}}{\pi \sigma_{k}^{2}} \exp \left(-\frac{\left|n_{r}\right|^{2}}{\sigma_{k}^{2}}\right)
$$

where $\sigma_{k}^{2}$ is the conditional variance given that $k$ impulses are affecting the receiver. It is calculated as

$$
\sigma_{k}^{2}=\sigma^{2} \beta_{k}
$$

\footnotetext{
${ }^{1}$ The overall channels, i.e., $\sqrt{g_{S D}} h_{S D}, \sqrt{g_{S R_{i}}} h_{S R_{i}}$, and $\sqrt{g_{R_{i} D}} h_{R_{i} D}$ are therefore modeled as independent zero-mean complex Gaussian random variables with different variances.
} 
where $\sigma^{2}$ denotes the mean variance of impulsive noise $n_{r}$ and is equal to $N_{0}$ and $\beta_{k}$ is given by

$$
\beta_{k}=\left(\frac{k A^{-1}+\Gamma}{1+\Gamma}\right) \text {. }
$$

In (6), $\Gamma$ is the Gaussian factor which is the ratio between the variance of background Gaussian component $\sigma_{g}^{2}$ and the variance of the impulsive component $\sigma_{i}^{2}$. Middleton Class A model is therefore characterized by two parameters, namely, impulsive index $A$ and Gaussian factor $\Gamma$. As $A$ decreases, i.e., $A \rightarrow 0$, noise becomes highly structured and more impulsive. On the contrary, if $A$ increases, i.e., $A \rightarrow \infty$, noise tends to Gaussian. Similarly, for small values of $\Gamma$, generated noise becomes more impulsive while it tends to Gaussian for large values of $\Gamma$. In our work, we assume that impulsive noise samples are temporally dependent during a transmission frame. This is well justified through experimental observations on impulsive noise [14]. As for spatial dimension, we consider both dependent and independent impulsive noise. Spatially dependent is applicable when the same set of interfering sources affects destination and relay nodes together. In this case, destination and relay nodes observe relatively the same distance to the interfering sources [16]. On the other hand, spatially independent case occurs if different sets of interfering sources affect destination and relay nodes.

\section{Transmission Model}

We assume Protocol II of [1], [5] which is the distributed SIMO (single-input multi-output) implementation. During the broadcasting phase (i.e., $P$ time slots), destination node $D$ and relays $R_{1}, \ldots, R_{M}$ are in receive mode. The received signals corrupted by fading and impulsive noise are first normalized at relay nodes to ensure unity of average energy. Then either repetition coding or space-time block coding is applied across these signals before they are forwarded to the destination in broadcasting phase which spans a duration of $L$ time slots. Let $E$ denote the average energy per time slot. Therefore, total energy consumed by source node $S$ in broadcasting phase (of $P$ time slots) is $P E$. On the other hand, in relaying phase (of $L$ time slots), $M$ relays consume a total energy of $L E$. Since we will deal with optimum power allocation in later sections, we also introduce the optimization parameters here. The first optimization parameter $\xi$ determines the ratio of power allocated between broadcasting and relaying phases. On the other hand, optimization parameters $\psi_{1}, \ldots, \psi_{M-1}$ are used to determine how the available relaying power is allocated among relay nodes.

Let $x_{1}, \ldots, x_{P}$ denote M-PSK (phase shift keying) modulation signals to be transmitted. The received signals at destination node $D$ and $i^{\text {th }}$ relay $R_{i}$ during $p^{\text {th }}$ time slot of the broadcasting phase are given respectively by

$$
\begin{gathered}
r_{D, p}=\sqrt{\widehat{\xi} E} h_{S D} x_{p}+n_{D, p}, \quad p=1, \ldots, P \\
r_{R_{i}, p}=\sqrt{g_{S R_{i}} \widehat{\xi} E} h_{S R_{i}} x_{p}+n_{R_{i}, p}
\end{gathered}
$$

where $\widehat{\xi}=((P+L) / P) \xi$. In (7)-(8), $n_{R_{i}, p}$ and $n_{D, p}$ represent the impulsive noise terms at relay and destination nodes.
After scaling by $\sqrt{g_{S R_{i}} \widehat{\xi} E+N_{0}}$ at corresponding relay nodes [5], [20], the received signals are applied to either a spacetime block encoder or simply sent through a repetition-based scheme [1].

First assume that space-time block codes (STBCs) of [22], [23] are employed across relay terminals. Let $\boldsymbol{Y}$ denote the space-time code matrix with size of $M \times L$ which consists of elements as linear functions of $x_{i, 1}, \ldots, x_{i, L}$ and/or $x_{i, 1}^{*}, \ldots, x_{i, L}^{*}$. Further, let $\boldsymbol{N}$ denote the $M \times L$ matrix for impulsive noise samples at relays. The received signal at destination during $(P+l)^{t h}$ time slot is

$$
r_{D, P+l}=\boldsymbol{\mu} \boldsymbol{y}_{l}+\boldsymbol{\lambda} \boldsymbol{n}_{l}+n_{D, P+l}, \quad l=1, \ldots, L
$$

where $\boldsymbol{y}_{l}$ is the $l^{\text {th }}$ column vector of $\boldsymbol{Y}$ and $\boldsymbol{n}_{l}$ is the $l^{\text {th }}$ column vector of $\boldsymbol{N}$ such that $i^{t h}$ element of $\boldsymbol{n}_{l} \in$ $\left\{n_{R_{i}, p},-n_{R_{i}, p}, n_{R_{i}, p}^{*},-n_{R_{i}, p}^{*}\right\}$ based on the form of code matrix $\boldsymbol{Y}$. In (9), $\boldsymbol{\mu}$ and $\boldsymbol{\lambda}$ are defined as

$$
\begin{aligned}
& \boldsymbol{\mu}=\left[\begin{array}{lll}
\varepsilon_{1} h_{S R_{1}}^{(*)} h_{R_{1} D} & \cdots & \varepsilon_{M} h_{S R_{M}}^{(*)} h_{R_{M} D}
\end{array}\right]_{1 \times M} \\
& \boldsymbol{\lambda}=\left[\begin{array}{lll}
v_{1} h_{R_{1} D} & \cdots & v_{M} h_{R_{M} D}
\end{array}\right]_{1 \times M}
\end{aligned}
$$

where $h_{S R_{i}}^{(*)} \in\left\{h_{S R_{i}}, h_{S R_{i}}^{*}\right\}$ and $\varepsilon_{i}$ and $v_{i}$ are given by

$$
\begin{gathered}
\varepsilon_{i}=\sqrt{\left(\widehat{\psi}_{i} g_{S R_{i}} g_{R_{i} D} \widehat{\xi}(1-\xi) E^{2}\right) /\left(g_{S R_{i}} \widehat{\xi} E+N_{0}\right)} \\
v_{i}=\sqrt{\left(\widehat{\psi}_{i} g_{R_{i} D}(1-\xi) E\right) /\left(g_{S R_{i}} \widehat{\xi} E+N_{0}\right)}
\end{gathered}
$$

with

$$
\widehat{\psi}_{i}=\left\{\begin{array}{l}
((P+L) / L) \psi_{i} \quad, i=1, \ldots, M-1 \\
((P+L) / L)\left(1-\sum_{i=1}^{M-1} \psi_{i}\right), i=M
\end{array} .\right.
$$

The received signals at destination node are normalized by $\Omega=\sqrt{1+\sum_{i=1}^{M} v_{i}^{2}}$ assuming so-called average power scaling (APS) [5], [24] which yields

$$
\widetilde{r}_{D, P+l}=\tilde{\boldsymbol{\mu}} \boldsymbol{y}_{l}+\widetilde{n}_{D, P+l}, \quad l=1, \ldots, L
$$

where we define $\widetilde{r}_{D, P+l}=r_{D, P+l} / \Omega, \widetilde{\boldsymbol{\mu}}=\boldsymbol{\mu} / \Omega$, and $\widetilde{n}_{D, P+l}=\left(\boldsymbol{\lambda} \boldsymbol{n}_{\boldsymbol{l}}+n_{D, P+l}\right) / \Omega$. To introduce a compact matrix form representation, we further define

$$
\begin{aligned}
& \boldsymbol{r}=\left[\begin{array}{lll}
r_{D, 1} \cdots r_{D, P} & \widetilde{r}_{D, P+1} \cdots \widetilde{r}_{D, P+L}
\end{array}\right]_{1 \times(P+L)} \\
& \boldsymbol{h}=\left[\sqrt{\widehat{\xi} E} h_{S D} \cdots \sqrt{\widehat{\xi} E} h_{S D} \tilde{\boldsymbol{\mu}}\right]_{1 \times(P+M)}
\end{aligned}
$$

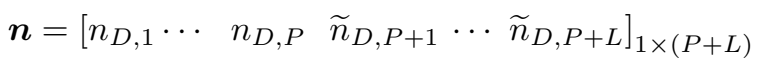

$$
\begin{aligned}
& \boldsymbol{X}=\left[\begin{array}{ll}
\boldsymbol{x}_{1} & \boldsymbol{x}_{2}
\end{array}\right]_{(P+M) \times(P+L)}
\end{aligned}
$$

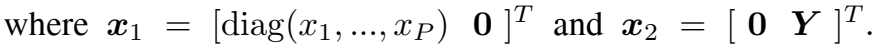
Then we have

$$
\boldsymbol{r}=\boldsymbol{h} \boldsymbol{X}+\boldsymbol{n}
$$

which describes the received signal for the transmission frame of $P+L$ slots.

If repetition-based cooperative scheme is used instead of space-time coding across the relay nodes, the received signal 
still takes the form of (20) where $\boldsymbol{r}, \boldsymbol{h}, \boldsymbol{n}$ and $\boldsymbol{X}$ are now defined as

$$
\begin{aligned}
& \boldsymbol{r}=\left[\begin{array}{ll}
r_{D, 1} \cdots r_{D, P} & r_{D, P+1} \cdots r_{D, P(M+1)}
\end{array}\right]_{1 \times P(M+1)} \\
& \boldsymbol{h}=\left[\sqrt{\widehat{\xi} E} h_{S D} \cdots \sqrt{\widehat{\xi} E} h_{S D} \quad \widetilde{\boldsymbol{\mu}} \cdots \widetilde{\boldsymbol{\mu}}\right]_{1 \times P(M+1)}
\end{aligned}
$$

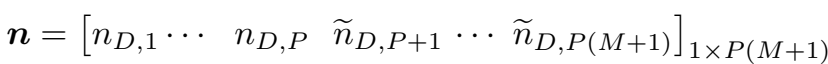

$$
\begin{aligned}
& \boldsymbol{X}=\left[\operatorname { d i a g } \left(x_{1}, \ldots, x_{P}, x_{1}, \ldots, x_{1}, \ldots\right.\right. \\
& \left.\left.\ldots, x_{P}, \ldots, x_{P}\right)\right]_{P(M+1) \times P(M+1)}
\end{aligned}
$$

with $\boldsymbol{\mu}=\left[\begin{array}{lll}\varepsilon_{1} h_{S R_{1}} h_{R_{1} D} & \cdots & \varepsilon_{M} h_{S R_{M}} h_{R_{M} D}\end{array}\right], \tilde{n}_{D, P+l}=$ $\left(\lambda_{n} n_{R_{n}, l}+n_{D, P+l}\right) / \Omega_{n}, \widetilde{\mu}_{i}=\mu_{i} / \Omega_{i}$ and $\Omega_{i}=\sqrt{1+v_{i}^{2}}$, $i \in\{1, \ldots, M\}, n=l+M(1-\lceil l / M\rceil)$ and $l \in\{1, \ldots, P M\}$. The received signal is then fed to an MDR [16] in which the detection rule is given by

$$
\widehat{\boldsymbol{X}}=\arg \min _{X}\|\boldsymbol{r}-\boldsymbol{h} \boldsymbol{X}\|^{2} .
$$

Although MDR is, in general, not optimal for impulsive noise environment, we use it as a practical low-complexity detection technique [16]. Further discussions on various types of receivers for impulsive noise can be found in the Appendix.

\section{DERIVATIONS OF PEP}

PEP constitutes the building block for the derivation of union bound to the error probability. Let $\boldsymbol{X}$ and $\widehat{\boldsymbol{X}}$ denote, respectively, the original transmitted codeword and decoded codeword. In our derivations, we consider both cases of spatially dependent and independent noise at destination and relay nodes. In both cases, noise samples are assumed to be temporally dependent during transmission frame.

\section{A. Spatially Dependent Noise}

First, we start with the spatially dependent case. The Euclidean distance between $\boldsymbol{X}$ and $\widehat{\boldsymbol{X}}$ conditioned on fading coefficients is given by

$$
d^{2}(\boldsymbol{X}, \widehat{\boldsymbol{X}})=\boldsymbol{h}(\boldsymbol{X}-\widehat{\boldsymbol{X}})(\boldsymbol{X}-\widehat{\boldsymbol{X}})^{H} \boldsymbol{h}^{H}
$$

Assume that the number of impulses affecting relays $R_{1}, \ldots, R_{M}$ and destination node $D$ is identical and denoted by Poisson random variable $C$. Considering all possible realizations of $C$, the conditional PEP is given by

$$
P(\boldsymbol{X}, \widehat{\boldsymbol{X}} \mid \boldsymbol{h})=\sum_{k=0}^{\infty} \alpha_{k} Q\left(\sqrt{d^{2}(\boldsymbol{X}, \widehat{\boldsymbol{X}}) / 2 \beta_{k} N_{0}}\right)
$$

where $\alpha_{k}$ and $\beta_{k}$ can be calculated through (3) and (6). Applying Chernoff bound, i.e., in (27) and taking expectation with respect to fading coefficients $\left|h_{S D}\right|^{2},\left|h_{S R_{1}}\right|^{2}, \ldots,\left|h_{S R_{M}}\right|^{2}$, which follow exponential distribution, the conditional PEP can be obtained as

$$
\begin{aligned}
P\left(\boldsymbol{X},\left.\widehat{\boldsymbol{X}}|| h_{R_{1} D}\right|^{2}, \ldots,\left|h_{R_{M} D}\right|^{2}\right) \\
\leq \sum_{k=0}^{\infty} \alpha_{k}\left(1+\frac{\widehat{\xi} \Delta E}{4 \beta_{k} N_{0}}\right)^{-1} \\
\quad \times \prod_{m=1}^{M}\left(1+\frac{\eta_{m} \varepsilon_{m}^{2}\left|h_{R_{m} D}\right|^{2} E}{4 \beta_{k} N_{0} \Omega^{2}}\right)^{-1}
\end{aligned}
$$

where $\Delta=\sum_{i=1}^{P}\left|x_{i}-\widehat{x}_{i}\right|^{2}$ and $\eta_{m}$ 's are the eigenvalues of STBC code matrix $\boldsymbol{Y}$. Further, taking expectation with respect to $\left|h_{R_{1} D}\right|^{2}, \ldots,\left|h_{R_{M} D}\right|^{2}$, which also follow exponential distribution, the unconditional PEP is obtained as

$$
\begin{aligned}
& P(\boldsymbol{X}, \widehat{\boldsymbol{X}}) \leq \sum_{k=0}^{\infty} \alpha_{k}\left(1+\frac{\widehat{\xi} \Delta E}{4 \beta_{k} N_{0}}\right)^{-1} \\
& \times \prod_{m=1}^{M}\left(\frac{\eta_{m} \varepsilon_{m}^{2}\left(E / N_{0}\right)}{4 \beta_{k} \Omega^{2}}\right)^{-1} \\
& \times \exp \left(\frac{4 \beta_{k} \Omega^{2}}{\eta_{m} \varepsilon_{m}^{2}\left(E / N_{0}\right)}\right) \Gamma\left(0, \frac{4 \beta_{k} \Omega^{2}}{\eta_{m} \varepsilon_{m}^{2}\left(E / N_{0}\right)}\right)
\end{aligned}
$$

where $\Gamma(.,$.$) is the upper incomplete Gamma function [25].$ The infinite summation in the derived PEP expression can be safely truncated to a finite summation without significant loss because probability of very large number of interferences is negligible and Poisson distribution approaches zero for large value of impulses.

Next, we will discuss the achievable diversity order based on the derived PEP. Diversity order for finite SNR values is defined as [26]

$$
d\left(E / N_{0}\right)=-E / N_{0}\left(\partial \log P(\boldsymbol{X} \rightarrow \widehat{\boldsymbol{X}}) / \partial\left(E / N_{0}\right)\right) .
$$

In low SNR region, i.e., $E / N_{0} \rightarrow 0$, an approximation of diversity order can be obtained as

$$
d \cong-\left[A_{1}+A_{2}+A_{3}+A_{4}\right]
$$

where

$$
\begin{gathered}
A_{1}=\sum_{m=1}^{M}\left(E / N_{0}\right)^{-1} \exp \left(-\frac{4 \Omega^{2} \beta_{0}}{\eta_{m} \varepsilon_{m}^{2}}\left(E / N_{0}\right)^{-1}\right) \\
\times \Gamma\left(0, \frac{4 \Omega^{2} \beta_{0}}{\eta_{m} \varepsilon_{m}^{2}}\left(E / N_{0}\right)^{-1}\right)^{-1} \\
A_{2}=-\beta_{0}\left(E / N_{0}\right)^{-2}\left(\sum_{m=1}^{M}\left(\frac{\eta_{m} \varepsilon_{m}^{2}}{4 \Omega^{2}}\right)^{-1}\right) \\
A_{3}=-M\left(E / N_{0}\right)^{-1} \\
A_{4}=-\left(\frac{\widehat{\xi} \Delta}{4 \beta_{0}+\widehat{\xi} \Delta E / N_{0}}\right) .
\end{gathered}
$$

For high SNR values, i.e. $E / N_{0}>30 \mathrm{~dB}$, an approximation of diversity order can be given as

$$
d \cong-\left(E / N_{0}\right)\left[A_{1}+A_{2}+A_{3}+A_{4}\right]
$$

where $A_{1}, A_{2}, A_{3}$ and $A_{4}$ are now calculated by replacing $\beta_{0}$ in (32)-(35) by $\beta_{1}$. For the limiting case of $E / N_{0} \rightarrow \infty$, it can be shown that $A_{1}$ and $A_{2}$ converge to zero. Replacing those in (36), we observe that the diversity order converges to $M+1$ which is the full spatial diversity for the $M$ relay system under consideration. However, it is important to note that due to the presence of Gamma function (as a result of cascaded channel structure over the relaying path) and summation term (as a result of impulsive noise) in (29), convergence to asymptotical diversity order is slower than we typically observe in a Rayleigh faded point-to-point multiantenna diversity link with AWGN.

We also note that the derived PEP expression includes the conventional Gaussian assumption as a special case. Recall that conditional variances of impulsive noise are given by 
$\beta_{k} N_{0}, k=0,1, \ldots$ As $\Gamma \rightarrow \infty, \beta_{k}$ 's converge to one. The summation of $\alpha_{k}$ in (29) over all possible values of $k$ therefore becomes equal to one. Consequently, (29) reduces to

$$
\begin{aligned}
& P(\boldsymbol{X}, \widehat{\boldsymbol{X}}) \leq\left(1+\frac{\widehat{\xi} \Delta E}{4 N_{0}}\right)^{-1} \\
& \times \prod_{m=1}^{M}\left(\frac{\eta_{m} \varepsilon_{m}^{2}\left(E / N_{0}\right)}{4 \Omega^{2}}\right)^{-1} \exp \left(\frac{4 \Omega^{2}}{\eta_{m} \varepsilon_{m}^{2}\left(E / N_{0}\right)}\right) \\
& \times \Gamma\left(0, \frac{4 \Omega^{2}}{\eta_{m} \varepsilon_{m}^{2}\left(E / N_{0}\right)}\right)
\end{aligned}
$$

which is the PEP expression for AWGN case.

\section{B. Spatially Independent Noise}

Now, we return our attention to the case where we have spatially independent noise samples. Assume that the number of impulses affecting relays $R_{1}, \ldots, R_{M}$ are statistically independent and denoted by Poisson random variables $C_{1}, \ldots, C_{M}$. On the other hand, $C_{M+1}$ is a Poisson random variable representing number of impulses affecting destination node $D$. Contrary to the single summation expression for spatially dependent case, the conditional PEP is now given by (38) (see the top of the next page). An approximate upper bound on conditional PEP can be obtained as in (39) (given at the top of the next page) by setting $\beta_{k_{1}}=\ldots=\beta_{k_{M+1}}=\Phi$, which denotes the average number of impulses affecting the destination and relay nodes during a transmission frame and is given by

$$
\Phi=\frac{L \sum_{m=1}^{M} \beta_{k_{m}}+(P+L) \beta_{k_{M+1}}}{(M+1) L+P}
$$

Taking expectations with respect to fading coefficients, we obtain the PEP as

$$
\begin{aligned}
& P(\boldsymbol{X}, \widehat{\boldsymbol{X}}) \widetilde{\leq} \sum_{k_{1}=0}^{\infty} \ldots \sum_{k_{i}=0}^{\infty} \ldots \sum_{k_{M+1}=0}^{\infty}\left(\prod_{n=1}^{M+1} \alpha_{k_{n}}\right) \\
& \times\left(1+\frac{\widehat{\xi} \Delta E}{4 \Phi N_{0}}\right)^{-1} \prod_{m=1}^{M}\left(\frac{\eta_{m} \varepsilon_{m}^{2}\left(E / N_{0}\right)}{4 \Phi \Omega^{2}}\right)^{-1} \\
& \times \exp \left(\frac{4 \Phi \Omega^{2}}{\eta_{m} \varepsilon_{m}^{2}\left(E / N_{0}\right)}\right) \Gamma\left(0, \frac{4 \Phi \Omega^{2}}{\eta_{m} \varepsilon_{m}^{2}\left(E / N_{0}\right)}\right)
\end{aligned}
$$

\section{Comparison of Exact and Derived PEPs}

To demonstrate the accuracy of derived PEPs, we present comparison of $(29) /(41)$ with the corresponding exact PEPs in Fig. 1. We consider two-relay Alamouti-based scheme, assume $\Theta_{1}=-30 \mathrm{~dB}$ and $\Theta_{2}=-30 \mathrm{~dB}$, and equal power allocation. We consider the shortest error event and compute exact PEP given by (27) through a Monte-Carlo simulation. From the figure, we observe that derived PEPs lie within $3 \mathrm{~dB}$ and $\sim 4 \mathrm{~dB}$ of the exact ones for $\mathrm{NG}$ and $\mathrm{HI}$ noise respectively. This discrepancy is mainly due to Chernoff bound used in the derivations.

\section{Repetition-based Cooperative Scheme}

For the repetition-based cooperative scheme [1], using similar steps as in the STBC-encoded case, we obtain unconditional PEP expression for the assumption of spatially

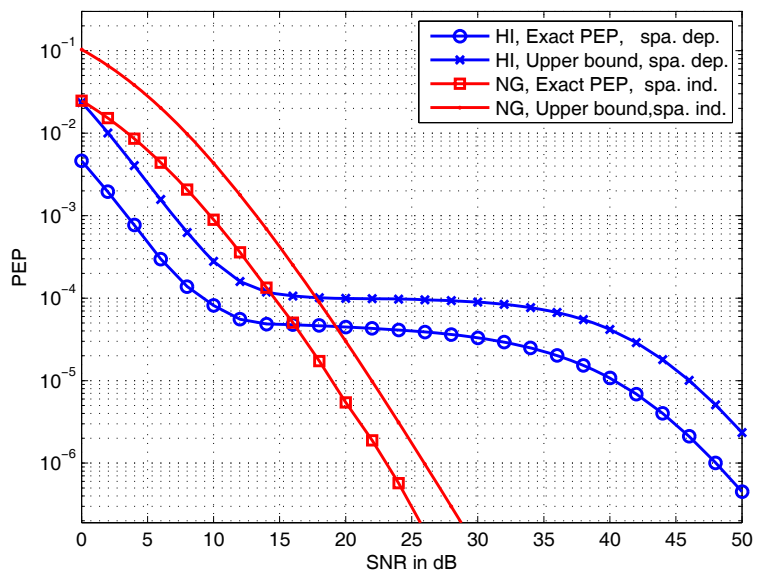

Fig. 1. Comparison of exact and derived PEPs over spatially dependent and independent impulsive noise.

dependent and independent noise, respectively, as

$$
\begin{aligned}
& P(\boldsymbol{X}, \widehat{\boldsymbol{X}}) \leq \sum_{k=0}^{\infty} \alpha_{k}\left(1+\frac{\widehat{\xi} \Delta E}{4 \beta_{k} N_{0}}\right)^{-1} \\
& \times \prod_{m=1}^{M}\left(\frac{\Delta \varepsilon_{m}^{2}\left(E / N_{0}\right)}{4 \beta_{k}\left(1+v_{m}^{2}\right)}\right)^{-1} \exp \left(\frac{4 \beta_{k}\left(1+v_{m}^{2}\right)}{\Delta \varepsilon_{m}^{2}\left(E / N_{0}\right)}\right) \\
& \times \Gamma\left(0, \frac{4 \beta_{k}\left(1+v_{m}^{2}\right)}{\Delta \varepsilon_{m}^{2}\left(E / N_{0}\right)}\right)
\end{aligned}
$$

$$
\begin{aligned}
& P(\boldsymbol{X}, \widehat{\boldsymbol{X}}) \widetilde{\leq} \sum_{k_{1}=0}^{\infty} \ldots \sum_{k_{i}=0}^{\infty} \ldots \sum_{k_{M+1}=0}^{\infty}\left(\prod_{n=1}^{M+1} \alpha_{k_{n}}\right) \\
& \times\left(1+\frac{\widehat{\xi} \Delta E}{4 \widetilde{\Phi} N_{0}}\right)^{-1} \prod_{m=1}^{M}\left(\frac{\Delta \varepsilon_{m}^{2}\left(E / N_{0}\right)}{4 \widetilde{\Phi}\left(1+v_{m}^{2}\right)}\right)^{-1} \\
& \times \exp \left(\frac{4 \widetilde{\Phi}\left(1+v_{m}^{2}\right)}{\Delta \varepsilon_{m}^{2}\left(E / N_{0}\right)}\right) \Gamma\left(0, \frac{4 \widetilde{\Phi}\left(1+v_{m}^{2}\right)}{\Delta \varepsilon_{m}^{2}\left(E / N_{0}\right)}\right)
\end{aligned}
$$

where $\widetilde{\Phi}=\left(P \sum_{m=1}^{M} \beta_{k_{m}}+P(M+1) \beta_{k_{M+1}}\right) /(P(1+$ $2 M))$.

\section{BER-Optimized Power Allocation}

Once PEP is obtained, we can obtain a union bound on BER using [27]

$$
P_{b} \leq \frac{1}{n} \sum_{X} P(\boldsymbol{X}) \sum_{\boldsymbol{X} \neq \widehat{\boldsymbol{X}}} q(\boldsymbol{X} \rightarrow \widehat{\boldsymbol{X}}) P(\boldsymbol{X} \rightarrow \widehat{\boldsymbol{X}})
$$

where $P(\boldsymbol{X})$ is the probability that codeword $\boldsymbol{X}$ is transmitted, $q(\boldsymbol{X} \rightarrow \widehat{\boldsymbol{X}})$ is the number of information bits in error if decoder decides in favor of $\widehat{\boldsymbol{X}}$, and $n$ is the number of information bits per transmission. Optimization of power allocation is based on minimizing the union bound on BER with respect to $\xi, \psi_{1}, \ldots, \psi_{M-1}$ subject to constraints $0 \leq \xi, \psi_{1}, \ldots, \psi_{M-1} \leq 1$. The minimization problem can be formulated as

$$
\min _{0 \leq \xi, \psi_{1}, \ldots, \psi_{M-1} \leq 1} \Lambda\left(\xi, \psi_{1}, \ldots, \psi_{M-1}\right)
$$

where $\Lambda\left(\xi, \psi_{1}, \ldots, \psi_{M-1}\right)$ is the objective function given by (44). The specific form of the objective function depends on the modulation scheme and coding employed across the relay terminals. For example, assume Alamouti-coded two-relay 


$$
\begin{aligned}
P(\boldsymbol{X}, \widehat{\boldsymbol{X}} \mid \boldsymbol{h}) & =\sum_{k_{1}=0}^{\infty} \ldots \sum_{k_{M+1}=0}^{\infty} \prod_{n=1}^{M+1} \alpha_{k_{n}} Q\left(\frac{d^{2}(\boldsymbol{X}, \widehat{\boldsymbol{X}})}{\sqrt{2\left(\widehat{\xi}\left|h_{S D}\right|^{2} \Delta E \beta_{k_{M+1}}+\sum_{m=1}^{M} \eta_{m}\left(\varepsilon_{m}^{2} / \Omega^{2}\right)\left|h_{S R_{m}}\right|^{2}\left|h_{R_{m} D}\right|^{2} \beta_{k_{m}}\right) N_{0}}}\right) \\
P(\boldsymbol{X}, \widehat{\boldsymbol{X}} \mid \boldsymbol{h}) & \approx \sum_{k_{1}=0}^{\infty} \ldots \sum_{k_{M+1}=0}^{\infty} \prod_{n=1}^{M+1} \alpha_{k_{n}} Q\left(\sqrt{\frac{\widehat{\xi}\left|h_{S D}\right|^{2} \Delta E+\sum_{m=1}^{M} \eta_{m}\left(\varepsilon_{m}^{2} / \Omega^{2}\right)\left|h_{S R_{m}}\right|^{2}\left|h_{R_{m} D}\right|^{2}}{2 \Phi N_{0}}}\right)
\end{aligned}
$$

scenario and 4-PSK modulation scheme with Gray mapping. For this scheme, the objective function takes the form of

$$
\begin{aligned}
\left.\Lambda\left(\xi, \psi_{1}\right)\right|_{Q P S K} & \leq \frac{1}{4} \sum_{i=1}^{16} \frac{1}{16}[4 \Psi(2)+12 \Psi(4) \\
& +12 \Psi(6)+4 \Psi(8)] \\
& =\Psi(2)+3 \Psi(4)+3 \Psi(6)+\Psi(8)
\end{aligned}
$$

where we use $\Psi(\Delta)$ to denote the derived PEP expressions, i.e., (29) for spatially dependent or (41) for spatially independent noise. The inner argument $\Delta$ represents the Euclidean distance, i.e., $\Delta=\left|x_{1}-\widehat{x}_{1}\right|^{2}+\left|x_{2}-\widehat{x}_{2}\right|^{2}$.

A closed-form solution for (45) is not available; however, this problem can be efficiently solved through numerical methods. In Tables I and II, we report optimization values through numerical methods for a two-relay system in HI and NG environments. We assume Alamouti-coding, 4-PSK modulation scheme, $\phi_{1}=\phi_{2}=\pi$, and $\Gamma=0.1 . A$ is chosen as $10^{-4}$ and 1 , respectively, for $\mathrm{HI}$ and NG noise. We investigate six distinct scenarios where we have different geometrical layouts for two relays:

- Scenario $1 \Theta_{1}=-30 \mathrm{~dB}, \Theta_{2}=-30 \mathrm{~dB}$,

- Scenario $2 \Theta_{1}=0 \mathrm{~dB}, \Theta_{2}=-30 \mathrm{~dB}$,

- Scenario $3 \Theta_{1}=30 \mathrm{~dB}, \Theta_{2}=-30 \mathrm{~dB}$,

- Scenario $4 \Theta_{1}=0 \mathrm{~dB}, \Theta_{2}=0 \mathrm{~dB}$,

- Scenario $5 \Theta_{1}=0 \mathrm{~dB}, \Theta_{2}=30 \mathrm{~dB}$,

- Scenario $6 \Theta_{1}=30 \mathrm{~dB}, \Theta_{2}=30 \mathrm{~dB}$.

In Table $\mathrm{I}^{2}$, we present the optimization values for $\mathrm{HI}$ noise. It is observed that these values differ significantly from [20] where optimization values for Gaussian noise are reported. We also notice that optimum values $\xi$ and $\psi_{1}$ follow a certain pattern based on the SNR range of interest. For scenario 1, optimum value $\xi$ remains almost constant for SNR values from $0 \mathrm{~dB}$ to $50 \mathrm{~dB}$. For scenarios 2 and $3, \xi$ is nearly constant in low SNR region followed by a transition region in the region of $15-20 \mathrm{~dB}$ and then convergence to a constant value. We see similar trends for optimum values of $\psi_{1}$. In Table II, we present optimization values for NG noise. Unlike the HI noise case, the values in general follow the trends reported in

\footnotetext{
${ }^{2}$ Since optimum power allocation did not yield any significant performance improvement (as confirmed through simulations), optimum values for scenarios 4-6 were omitted from these tables.
}

TABLE I

OPTIMUM POWER ALLOCATION VALUES FOR HI CHANNEL

\begin{tabular}{|c|c|c|c|c|c|c|}
\hline \multirow{2}{*}{$\begin{array}{c}\text { SNR } \\
{[\mathrm{dB}]}\end{array}$} & \multicolumn{2}{|c|}{ Scenario 1} & \multicolumn{2}{c|}{ Scenario 2} & \multicolumn{2}{c|}{ Scenario 3 } \\
\cline { 2 - 7 } & $\xi$ & $\psi_{1}$ & $\xi$ & $\psi_{1}$ & $\xi$ & $\psi_{1}$ \\
\hline \hline 0 & 0.9726 & 0.5000 & 0.7933 & 0.9919 & 0.9719 & 0.0001 \\
\hline 5 & 0.9750 & 0.5000 & 0.7785 & 0.9946 & 0.7469 & 0.9957 \\
\hline 10 & 0.9758 & 0.5000 & 0.7712 & 0.9954 & 0.7205 & 0.9970 \\
\hline 15 & 0.9771 & 0.5000 & 0.7922 & 0.9943 & 0.7585 & 0.9957 \\
\hline 20 & 0.9783 & 0.5000 & 0.9599 & 0.7276 & 0.9498 & 0.8308 \\
\hline 25 & 0.9785 & 0.5000 & 0.9777 & 0.1161 & 0.9777 & 0.1179 \\
\hline 30 & 0.9787 & 0.5000 & 0.9798 & 0.0071 & 0.9798 & 0.0001 \\
\hline 35 & 0.9787 & 0.5000 & 0.9807 & 0.0001 & 0.9807 & 0.0001 \\
\hline 40 & 0.9784 & 0.5000 & 0.9810 & 0.0001 & 0.9810 & 0.0001 \\
\hline 45 & 0.9779 & 0.5000 & 0.8078 & 0.9941 & 0.9808 & 0.0001 \\
\hline 50 & 0.9772 & 0.5000 & 0.7897 & 0.9950 & 0.9723 & 0.5060 \\
\hline
\end{tabular}

TABLE II

OPTIMUM POWER ALLOCATION VALUES FOR NG CHANNEL

\begin{tabular}{|c|c|c|c|c|c|c|}
\hline \multirow{2}{*}{$\begin{array}{c}\text { SNR } \\
{[\mathrm{dB}]}\end{array}$} & \multicolumn{2}{|c|}{ Scenario 1 } & \multicolumn{2}{c|}{ Scenario 2} & \multicolumn{2}{c|}{ Scenario 3 } \\
\cline { 2 - 7 } & $\xi$ & $\psi_{1}$ & $\xi$ & $\psi_{1}$ & $\xi$ & $\psi_{1}$ \\
\hline \hline 0 & 0.9741 & 0.5000 & 0.9771 & 0.0001 & 0.9771 & 0.0001 \\
\hline 5 & 0.9764 & 0.5000 & 0.8118 & 0.9927 & 0.9795 & 0.0001 \\
\hline 10 & 0.9767 & 0.5000 & 0.7912 & 0.9947 & 0.9800 & 0.0001 \\
\hline 15 & 0.9765 & 0.5000 & 0.7787 & 0.9953 & 0.7499 & 0.9963 \\
\hline 20 & 0.9762 & 0.5000 & 0.7707 & 0.9956 & 0.7213 & 0.9971 \\
\hline 25 & 0.9760 & 0.5000 & 0.7655 & 0.9957 & 0.7051 & 0.9975 \\
\hline 30 & 0.9758 & 0.5000 & 0.7620 & 0.9958 & 0.6953 & 0.9976 \\
\hline 35 & 0.5009 & 0.5000 & 0.7597 & 0.9959 & 0.6890 & 0.9977 \\
\hline 40 & 0.5000 & 0.5000 & 0.5010 & 0.5006 & 0.9644 & 0.7175 \\
\hline 45 & 0.5000 & 0.5000 & 0.5000 & 0.5000 & 0.5000 & 0.5000 \\
\hline 50 & 0.5000 & 0.5000 & 0.5000 & 0.5000 & 0.5000 & 0.5000 \\
\hline
\end{tabular}

[20]. In scenario 1 where both relays are close to destination, optimized $\xi$ is about 0.97 for SNR $<30 \mathrm{~dB}$ then converges to 0.5 . For scenarios 2 and 3 , optimized $\xi$ reaches first 0.76 and 0.69 respectively at $35 \mathrm{~dB}$ and then converges to 0.5 .

As a final note in this section, we should emphasize that calculation of optimization values depend on noise parameters $A$ and $\Gamma$. There is, however, already an extensive literature on the estimation of impulsive noise parameters, see e.g., [28]-[30] and the references therein. Once these estimates are available, the power allocation parameters can be obtained and 


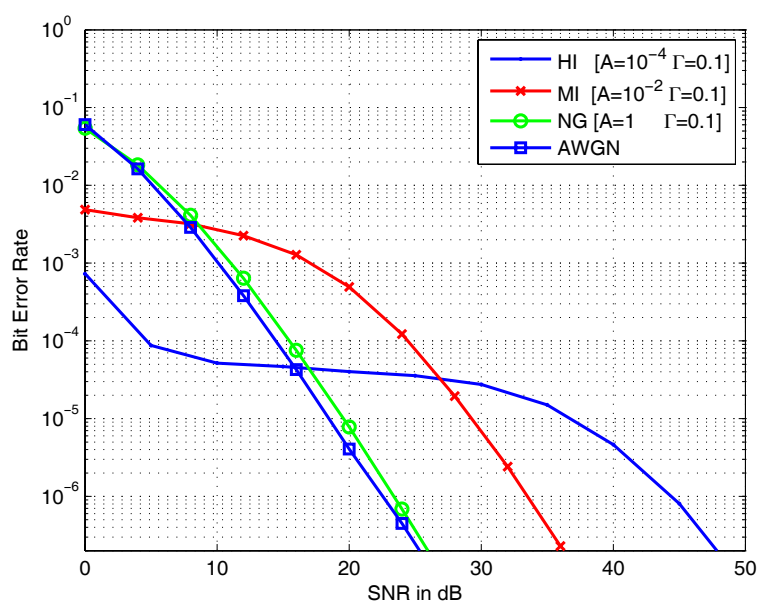

Fig. 2. Performance of Alamouti-coded two-relay scheme over Rayleigh fading channel in the presence of $\mathrm{HI}, \mathrm{MI}$, and NG noise for scenario 1.

then fed back to the nodes to adjust their powers accordingly. In practice, quantized versions of the estimates can be sent through the control channels.

\section{Simulation Results And Discussion}

In this section, we present Monte Carlo simulation results to demonstrate the error rate performance of multi-relay system under impulsive noise assuming equal and optimum power allocation. We consider a two-relay cooperative system where the relays either employ Alamouti or repetition coding. To ensure a fair comparison in throughput rate, Alamouti and repetition-based schemes are simulated with 4-PSK and 8PSK schemes, respectively.

In Fig. 2, we present the simulated BER performance of the Alamouti-based scheme over Rayleigh fading channel in the presence of spatially dependent impulsive noise with various impulsiveness degrees. Specifically, we consider HI noise with $A=10^{-4}$, moderate impulsive (MI) noise with $A=10^{-2}$ and NG noise with $A=1$. In all three noise environments, we have $\Gamma=0.1$. The performance over Rayleigh fading channel in the presence of AWGN is also included as a benchmark. We first consider scenario 1 where both relays are located close to destination. As observed from Fig. 2, the performance under impulsive noise dramatically differs from that of AWGN.

The distinguishing characteristics of system behavior in HI noise is three-region performance; BER curve first decreases linearly with SNR, followed by a flat-region, finally decreasing again with increasing SNR. As the impulsive index gets larger, the flat-region tends to appear early, i.e., in the lower SNR region. Once the impulsive index is sufficiently large (i.e., channel tends to Gaussian), it disappears. On a log-log scale, through the calculation of BER slopes, we can also check the diversity orders. In higher SNR values (after the flat region), the diversity orders in HI, MI, NG, and AWGN channels are respectively, $2.12,2.56,2.62$, and 2.63 . These can be also confirmed through (36). It is obvious that full diversity of three is not extracted under the simulated SNR range due to the harshness of the channel. This can be further observed in Fig. 3 where we plot finite diversity order given by (30)

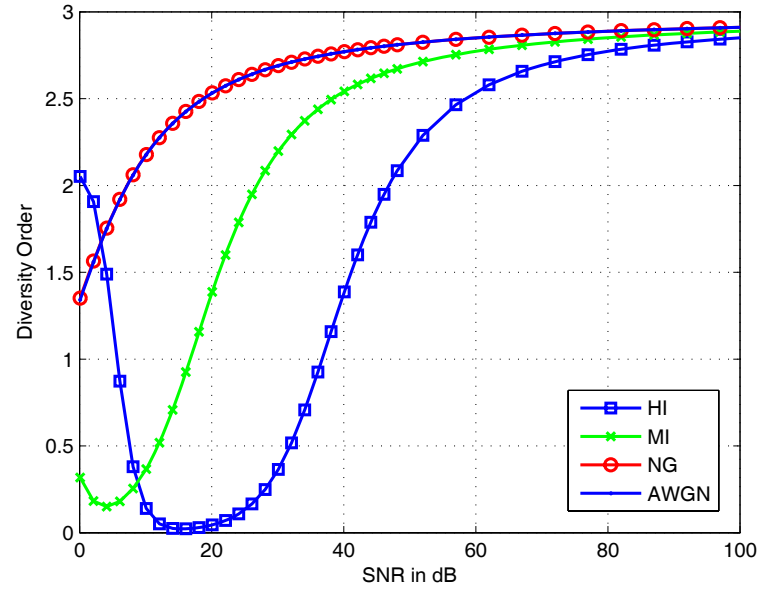

Fig. 3. Diversity order Alamouti-coded two-relay scheme over Rayleigh fading channels in the presence of $\mathrm{HI}, \mathrm{MI}$, and NG noise.

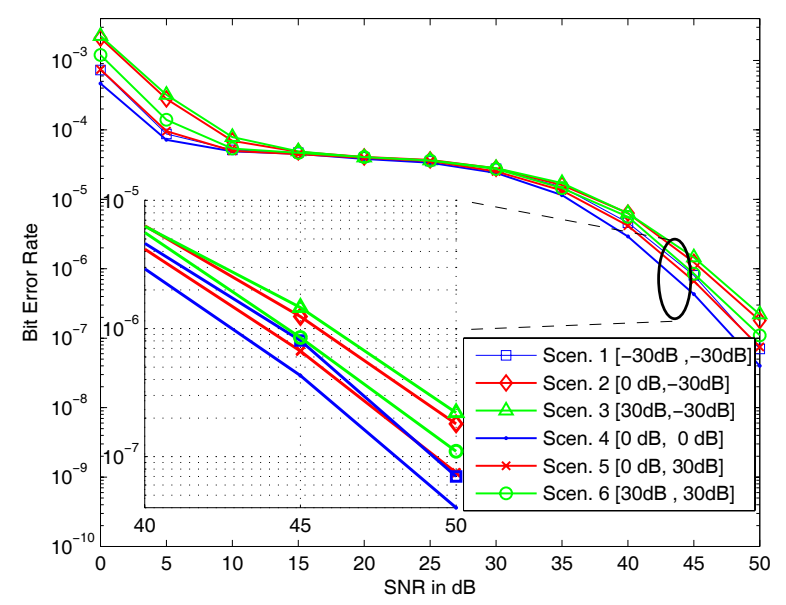

Fig. 4. BER performance of Alamouti-encoded two-relay scheme for various relay locations.

based on the derived PEP expressions. The convergence to full spatial diversity order of three takes place for higher SNR values.

In an attempt to demonstrate the effects of relays' location, we illustrate in Fig. 4 the performance of the Alamouti-based scheme for scenarios 1-6. We assume HI noise with $A=10^{-4}$ and $\Gamma=0.1$. We observe from Fig. 4 that the same diversity order is obtained irrespective of the location of relays. The best performance is however attained in scenario 4 where both relay nodes are located in the middle between source and destination nodes. The worst performance is observed in scenario 3 where one of the relays is closer to source node and the other relay is closer to destination node. It is interesting to note that performance comparisons among scenarios highly differ based on the coding employed across relay nodes. As illustrated in Fig. 5, repetition code based scheme yields the best performance in scenario 1 where both relays are located close to destination node. A further comparison between Figs. 4 and 5 reveals that, in scenarios 13 , repetition-based cooperative scheme outperforms Alamouti- 
t]

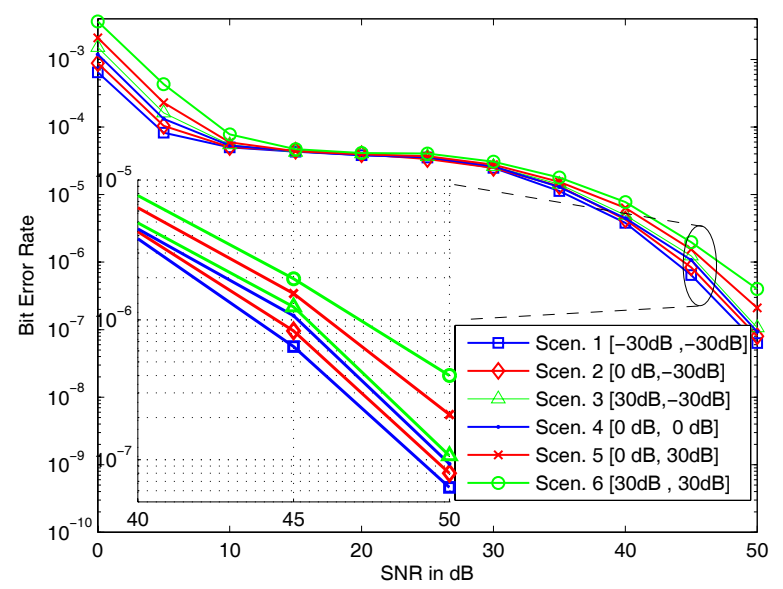

Fig. 5. BER performance of repetition-based coded scheme for various relay locations.

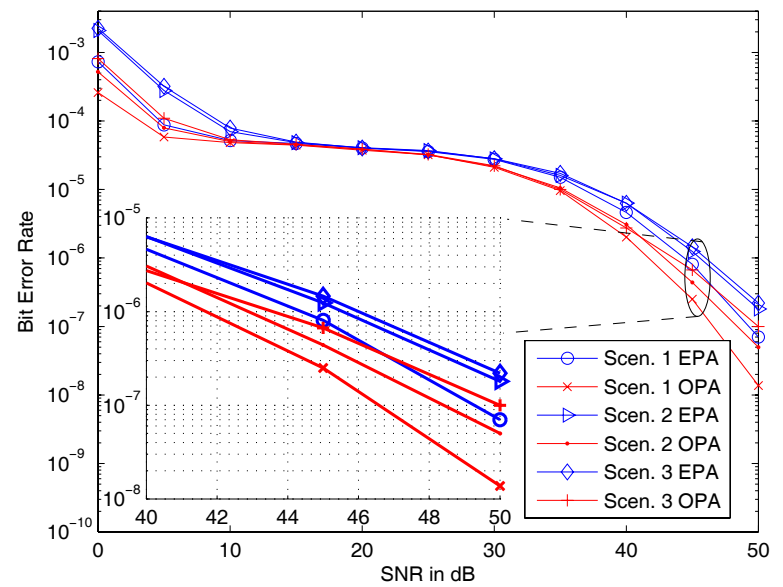

Fig. 6. Performance comparison of OPA and EPA in HI noise.

based scheme by $0.5-1 \mathrm{~dB}$ at a target BER of $10^{-6}$. On the other hand, scenarios 4-6 become favorable for Alamoutibased scheme which outperforms repetition-based cooperative scheme by $2-2.5 \mathrm{~dB}$ at $\mathrm{BER}=10^{-6}$.

In Figs. 6 and 7, we demonstrate the performance gains achieved through optimum power allocation (OPA) for Alamouti-based scheme. We assume HI and NG noise in Fig. 6 and Fig. 7, respectively. In HI noise, we observe gains in the range of $2-3 \mathrm{~dB}$ at target $\mathrm{BER}=10^{-6}$ depending on the relay locations. The gain is less as we move closer to the flat region. For example, at $\mathrm{BER}=10^{-5}$, gains are in the range of 1$2 \mathrm{~dB}$. On the other hand, in NG noise we observe performance improvements of $2.5-5 \mathrm{~dB}$ at $\mathrm{BER}=10^{-5}$. It turns out that OPA is more rewarding as the noise approaches to Gaussian nature.

So far we have assumed that noise samples are spatially dependent. As earlier discussed, this can be justified when the same set of interfering sources affects destination and relay nodes together. On the other hand, when different sets of interfering sources affect destination and relay nodes, we need to consider spatially independent noise. In Fig. 8, we demonstrate the effect of spatial independence on the BER

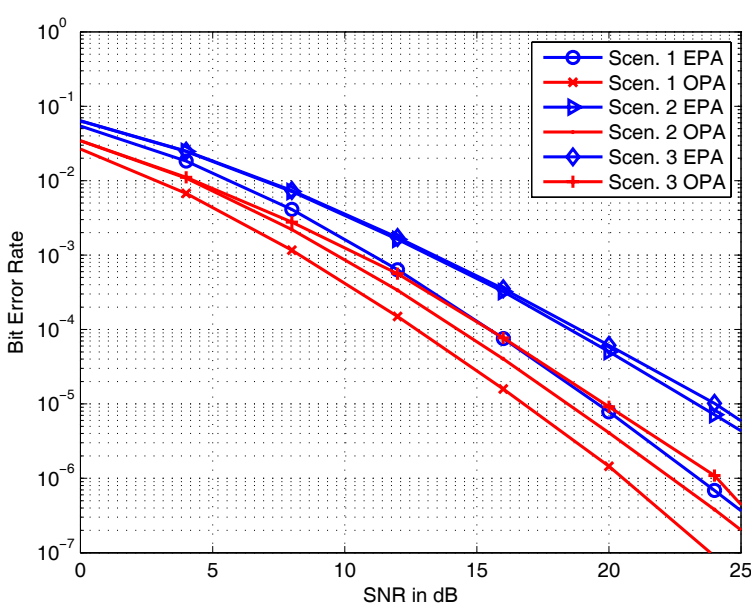

Fig. 7. Performance comparison of OPA and EPA in NG channel.

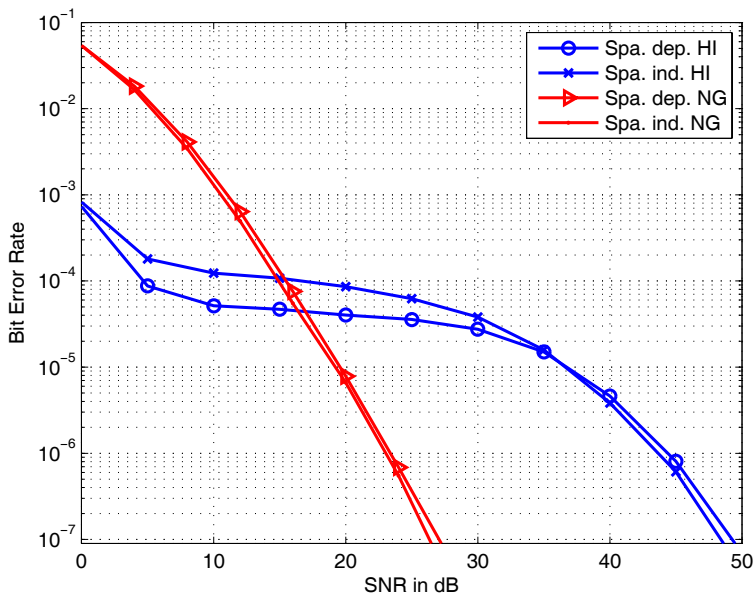

Fig. 8. Performance of MDR over spatially dependent and independent impulsive noise.

performance. If the noise is $\mathrm{HI}$ and SNR values are sufficiently small (i.e., $\mathrm{SNR}<35 \mathrm{~dB}$ ), performance in spatially dependent noise is better than that over spatially independent case. In higher SNR region, this flips over and performance over spatially independent noise becomes better. On the other hand, both cases exhibit similar performance in NG noise, regardless of SNR region.

To demonstrate the potential performance improvements of GAR and MAP receivers (see Appendix) over spatially independent impulsive noise, we present further simulations in Fig. 9 comparing their performance with that of MDR. From this figure, we observe that GAR and MAP receivers take advantage of additional knowledge on noise parameters, and are able to remove the flat region observed in the case of MDR deployment, thereby significantly outperforming MDR in HI noise. On the other hand, in NG noise, the performance improvement is much smaller.

\section{CONCLUSION}

In this paper, we have studied the performance of amplifyand-forward relaying schemes in the presence of impulsive 


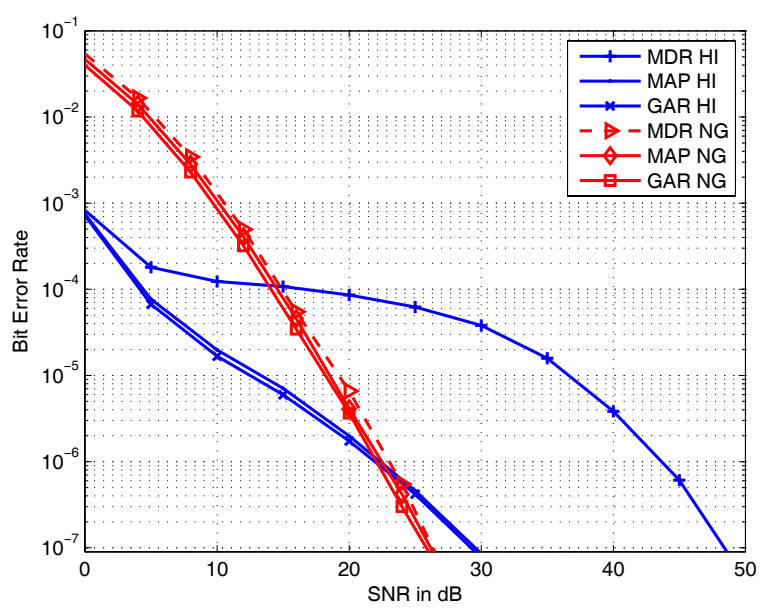

Fig. 9. Performance comparison of MDR, MAP, and GAR for spatially independent impulsive noise.

noise. For a multi-relay scheme, we have derived PEP expressions assuming Rayleigh fading channel, Middleton Class A noise, and MDR deployment. We have considered both spatially dependent and independent interferers. The derived expressions include conventional AWGN as a special case and can be considered as a generalization of existing results in the literature. Through PEP, we have demonstrated that full spatial diversity can be extracted in HI noise environment for sufficiently high SNR values. The performance of cooperative system in lower SNR regions however depends on the impulsive nature of noise and different diversity orders dominate the performance in different ranges of SNRs. Based on the optimization of a union bound on BER, we have further proposed power allocation rules for performance improvement. Performance gains up to $5 \mathrm{~dB}$ at $\mathrm{BER}=10^{-5}$ have been observed depending on the relay geometry and impulsiveness degree.

\section{APPENDIX}

In the following, we derive GAR and MAP detection rules for Alamouti-based scheme under the assumption of temporally-dependent impulsive noise. We consider both spatially-dependent and spatially-independent impulsive noise cases.

\section{A. MAP detection rule for spatially independent noise}

We first derive MAP detection rule for spatially independent case. Define $\boldsymbol{B}$ as

$\boldsymbol{B}=\left[C_{1} C_{2} \frac{\left(v_{1}^{2} C_{2}+v_{2}^{2} C_{3}+C_{1}\right)}{\Omega^{2}} \frac{\left(v_{1}^{2} C_{2}+v_{2}^{2} C_{3}+C_{1}\right)}{\Omega^{2}}\right]$

where $C_{1}, C_{2}$, and $C_{3}$ are Poisson random variables with $P\left(C_{i}=\sigma_{k_{i}}^{2}\right)=\left(e^{-A} A^{k_{i}}\right) / k_{i}$ !. Probability density function of $\boldsymbol{r}$ given $\boldsymbol{X}, \boldsymbol{h}$, and $\boldsymbol{B}$ is

$$
p(\boldsymbol{r} \mid \boldsymbol{X}, \boldsymbol{h}, \boldsymbol{B})=\frac{1}{\pi^{4} \operatorname{det} \boldsymbol{C}} \quad e^{-(\boldsymbol{r}-\boldsymbol{h} \boldsymbol{X})^{H} \boldsymbol{C}^{-1}(\boldsymbol{r}-\boldsymbol{h} \boldsymbol{X})}
$$

where $\boldsymbol{C}=\operatorname{diag}(\boldsymbol{B}(1), \boldsymbol{B}(2), \boldsymbol{B}(3), \boldsymbol{B}(4))$ is the covariance matrix of $\boldsymbol{r}$. Taking expectation with respect to $C_{1}, C_{2}$, and $C_{3}$, we obtain $p(\boldsymbol{r} \mid \boldsymbol{X}, \boldsymbol{h})$ which readily yields MAP rule as

$$
\begin{aligned}
\widehat{\boldsymbol{X}}=\underset{X}{\operatorname{argmax}} & p(\boldsymbol{r} \mid \boldsymbol{X}, \boldsymbol{h}) \\
=\underset{X}{\operatorname{argmax}} & \sum_{k_{1}=0}^{\infty} \sum_{k_{2}=0}^{\infty} \sum_{k_{3}=0}^{\infty} \frac{\alpha_{k_{1}} \alpha_{k_{2}} \alpha_{k_{3}}}{\pi^{4}\left(\sigma_{k_{1}}^{2}\right)^{2}\left(v_{1}^{2} \sigma_{k_{2}}^{2}+v_{2}^{2} \sigma_{k_{3}}^{2}+\sigma_{k_{1}}^{2}\right)^{2}} \\
& \times \exp \left(-\frac{\left|r_{1}-\boldsymbol{h} \boldsymbol{X}_{1}\right|^{2}+\left|r_{2}-\boldsymbol{h} \boldsymbol{X}_{2}\right|^{2}}{\sigma_{k_{1}}^{2}}\right) \\
& \times \exp \left(-\frac{\left|r_{3}-\boldsymbol{h} \boldsymbol{X}_{3}\right|^{2}+\left|r_{4}-\boldsymbol{h} \boldsymbol{X}_{4}\right|^{2}}{\left(v_{1}^{2} \sigma_{k_{2}}^{2}+v_{2}^{2} \sigma_{k_{3}}^{2}+\sigma_{k_{1}}^{2}\right) / \Omega^{2}}\right)
\end{aligned}
$$

where $\alpha_{k_{i}}=\left(e^{-A} A^{k_{i}}\right) / k_{i} !, \sigma_{k_{i}}^{2}=\sigma^{2}\left(k_{i} A^{-1}+\Gamma\right) /(1+\Gamma)$, $r_{i}$ is the $i^{t h}$ element of $\boldsymbol{r}$, and $\boldsymbol{X}_{i}$ is $i^{\text {th }}$ column vector of $\boldsymbol{X}$ respectively.

\section{B. MAP detection rule for spatially dependent noise}

For spatially dependent case, we have (47) in which $\boldsymbol{B}$ now takes the form of $\boldsymbol{B}=\left[\begin{array}{llll}C_{1} & C_{1} & C_{1} & C_{1}\end{array}\right]$ with $\boldsymbol{C}=$ $C_{1} \boldsymbol{I}_{4}$. Taking expectation with respect to $C_{1}$, we have

$$
p(\boldsymbol{r} \mid \boldsymbol{X}, \boldsymbol{h})=\sum_{k_{1}=0}^{\infty} \frac{\alpha_{k_{1}}}{\left(\pi \sigma_{k_{1}}^{2}\right)^{4}} e^{-\left(\sum_{i=1}^{4}\left|r_{i}-\boldsymbol{h} \boldsymbol{X}_{i}\right|^{2}\right) / \sigma_{k_{1}}^{2}} .
$$

Note that index of summation above is independent from $\boldsymbol{X}, \alpha_{k_{1}}$ is a scalar, and exponential function is a monotonic function. Therefore, MAP detection rule can be obtained as

$$
\widehat{\boldsymbol{X}}=\underset{X}{\operatorname{argmin}}\left(\sum_{i=1}^{4}\left|r_{i}-\boldsymbol{h} \boldsymbol{X}_{i}\right|^{2}\right) \text {. }
$$

We notice that MAP receiver coincides with MDR for temporally and spatially dependent noise where we have $\sum_{k_{1}=0}^{\infty} \alpha_{k_{1}}=1$.

\section{GAR detection rule for spatially independent noise}

\section{Define $K$ as}

$$
\boldsymbol{K}=\left[\sigma_{1}^{2} \sigma_{1}^{2} \frac{\left(v_{1}^{2} \sigma_{2}^{2}+v_{2}^{2} \sigma_{3}^{2}+\sigma_{1}^{2}\right)}{\Omega^{2}} \frac{\left(v_{1}^{2} \sigma_{2}^{2}+v_{2}^{2} \sigma_{3}^{2}+\sigma_{1}^{2}\right)}{\Omega^{2}}\right]
$$

where $\sigma_{1}^{2}, \sigma_{2}^{2}$, and $\sigma_{3}^{2}$ are known conditional variances at destination, first relay $\left(R_{1}\right)$ and second relay $\left(R_{2}\right)$ nodes respectively. We have

$$
p(\boldsymbol{r} \mid \boldsymbol{X}, \boldsymbol{h}, \boldsymbol{K})=\frac{1}{\pi^{4} \operatorname{det} \boldsymbol{C}} \quad e^{-(\boldsymbol{r}-\boldsymbol{h} \boldsymbol{X})^{H} \boldsymbol{C}^{-1}(\boldsymbol{r}-\boldsymbol{h} \boldsymbol{X})}
$$

where $\boldsymbol{C}=\operatorname{diag}(\boldsymbol{K}(1), \boldsymbol{K}(2), \boldsymbol{K}(3), \boldsymbol{K}(4))$. Hence, GAR detection rule is

$$
\widehat{\boldsymbol{X}}=\underset{X}{\operatorname{argmax}} \frac{1}{\pi^{4} \operatorname{det} \boldsymbol{C}} \quad e^{-(\boldsymbol{r}-\boldsymbol{h} \boldsymbol{X})^{H} \boldsymbol{C}^{-1}(\boldsymbol{r}-\boldsymbol{h} \boldsymbol{X})} .
$$

Noting exponential function is a monotonic function and ignoring the terms which do not affect maximization, we have

$$
\begin{aligned}
\widehat{\boldsymbol{X}}=\underset{X}{\operatorname{argmin}}\left(\frac{\left|r_{1}-\boldsymbol{h} \boldsymbol{X}_{1}\right|^{2}+\left|r_{2}-\boldsymbol{h} \boldsymbol{X}_{2}\right|^{2}}{\sigma_{1}^{2}}\right. \\
\left.+\frac{\left|r_{3}-\boldsymbol{h} \boldsymbol{X}_{3}\right|^{2}+\left|r_{4}-\boldsymbol{h} \boldsymbol{X}_{4}\right|^{2}}{\left(v_{1}^{2} \sigma_{2}^{2}+v_{2}^{2} \sigma_{3}^{2}+\sigma_{1}^{2}\right) / \Omega^{2}}\right)
\end{aligned}
$$




\section{GAR detection rule for spatially dependent noise}

In a similar approach to spatially independent case, we can derive GAR detection rule for spatially dependent case by substituting $\boldsymbol{K}=\left[\begin{array}{llll}\sigma_{1}^{2} & \sigma_{1}^{2} & \sigma_{1}^{2} & \sigma_{1}^{2}\end{array}\right]$ in (51). This yields

$$
\widehat{\boldsymbol{X}}=\underset{X}{\operatorname{argmin}}\left(\left(\sum_{i=1}^{4}\left|r_{i}-\boldsymbol{h} \boldsymbol{X}_{i}\right|^{2}\right) / \sigma_{1}^{2}\right)
$$

which is equivalent to MDR.

\section{REFERENCES}

[1] J. N. Laneman and G. W. Wornell, "Distributed space-time-coded protocols for exploiting cooperative diversity in wireless networks," IEEE Trans. Inform. Theory, vol. 49, no. 10, pp. 2415-2425, Oct. 2003.

[2] J. N. Laneman, D. N. C. Tse, and G. W. Wornell, "Cooperative diversity in wireless networks: efficient protocols and outage behavior," IEEE Trans. Inform. Theory, vol. 50, no. 12, pp. 3062-3080, Dec. 2004.

[3] A. Sendonaris, E. Erkip, and B. Aazhang, "User cooperation diversitypart I: system description," IEEE Trans. Commun., vol. 51, no. 11, pp. 1927-1938, Nov. 2003

[4] A. Sendonaris, E. Erkip, and B. Aazhang, "User cooperation diversitypart II: implementation aspects and performance analysis," IEEE Trans. Commun., vol. 51, no. 11, pp. 1939-1948, Nov. 2003.

[5] R. U. Nabar, H. Bolcskei, and F. W. Kneubuhler, "Fading relay channels: performance limits and space-time signal design," IEEE J. Select. Areas Commun., vol. 22, no. 6, pp. 1099-1109, Aug. 2004.

[6] E. C. Van der Meulen, "Three-terminal communication channels," Adv. Appl. Prob., vol. 3, no. 1, pp. 120-154, 1971.

[7] F. H. P. Fitzek and M. D. Katz (eds.), Cooperation in Wireless Networks: Principles and Applications Real Egoistic Behavior is to Cooperate!. Dordrecht, Netherlands: Springer, 2006.

[8] M. Uysal (ed.), Cooperative Communications for Improved Wireless Network Transmission: Frameworks for Virtual Antenna Array Applications. IGI Global, 2009.

[9] E. N. Skomal, "The range and frequency dependence of VHF-UHF manmade radio noise in and above metropolitan areas," IEEE Trans. Veh. Technol., vol. 19, no. 2, pp. 213-221, May 1970.

[10] K. L. Blackard, T. S. Rappaport, and C. W. Bostian, "Measurements and models of radio frequency impulsive noise for indoor wireless communications," IEEE J. Select. Areas Commun., vol. 11, no. 7, pp. 991-1001, Sept. 1993.

[11] M. S. Kuran and T. Tugcu, "A survey on emerging broadband wireless access technologies," Computer Networks, vol. 51, no. 11, pp. 30133046, Jan. 2007.

[12] D. Middleton, "Statistical-physical models of electromagnetic interference," IEEE Trans. Electromagn. Compat., vol. EMC-19, pp. 106-127, Aug. 1977.

[13] D. Middleton, "Non-Gaussian noise models in signal processing for telecommunications: new methods and results for class A and class B noise models," IEEE Trans. Inform. Theory, vol. 45, no. 4, pp. 11291149, May 1999.

[14] P. A. Delaney, "Signal detection in multivariate class A interference," IEEE Trans. Commun., vol. 43, no. 2, pp. 365-373, Feb. 1995.

[15] R. Haring and A. J. H. Vinck, "Performance bounds for optimum and suboptimum reception under class A impulsive noise," IEEE Trans. Commun., vol. 50, no.7, pp. 1130-1136, July 2002.

[16] P. Gao and C. Tepedelenlioglu, "Space-time coding over fading channels with impulsive noise," IEEE Trans. Wireless Commun., vol. 6, no. 1, pp. 220-229, Jan. 2007.

[17] A. Spaulding and D. Middleton, "Optimum reception in an impulsive interference environment-part I: coherent detection," IEEE Trans. Commun., vol. 25, no. 9, pp. 910-923, Sept. 1977.

[18] A. Spaulding and D. Middleton, "Optimum reception in an impulsive interference environment-part II: incoherent reception," IEEE Trans. Commun., vol. 25, no. 9, pp. 924-934, Sept. 1977.

[19] C. Tepedelenlioglu and P. Gao, "On diversity reception over fading channels with impulsive noise," IEEE Trans. Veh. Technol., vol. 54, no. 6, pp. 2037-2047, Nov. 2005.

[20] M. M. Fareed and M. Uysal, "BER-optimized power allocation for fading relay channels," IEEE Trans. Wireless Commun., vol. 7, no. 6, pp. 2350-2359, June 2008.
[21] D. Middleton, "Canonical and quasi-canonical probability models of class A interference," IEEE Trans. Electromagn. Compat., vol. EMC25, no. 2, pp. 76-106, May 1983.

[22] V. Tarokh, H. Jafarkhani, and A. R. Calderbank, "Space-time block codes from orthogonal designs," IEEE Trans. Inform. Theory, vol. 45, no. 5, pp. 1456-1467, July 1999.

[23] Y. Jing and H. Jafarkhani, "Using Orthogonal and quasi-orthogonal designs in wireless relay networks," IEEE Trans. Inform. Theory, vol. 53, no. 11, pp. 4106-4118, Nov. 2007.

[24] H. Mheidat and M. Uysal, "Impact of receive diversity on the performance of amplify-and-forward relaying under APS and IPS power constraints," IEEE Commun. Lett., vol. 10, no. 6, pp. 468-470, June 2006.

[25] I. S. Gradshteyn and I. M. Ryzhik, Table of Integrals, Series, and Products. Academic Press, 5th edition, 1994.

[26] R. Narasimhan, "Finite-SNR diversity-multiplexing tradeoff for correlated Rayleigh and Rician MIMO channels," IEEE Trans. Inform. Theory, vol. 52, no. 9, pp. 3965-3979, Sept. 2006.

[27] S. Benedetto and E. Biglieri, Principles of Digital Transmission with Wireless Applications. Norwell, MA: Kluwer Academic Publishers, 1999.

[28] S. M. Zabin and H. V. Poor, "Parameter estimation for Middleton Class A interference processes," IEEE Trans. Commun., vol. 37, no. 10, pp. 1042-1051, Oct. 1989

[29] S. M. Zabin and H. V. Poor, "Recursive algorithms for identification of impulse noise channels," IEEE Trans. Inform. Theory, vol. 36, no. 3, pp. 559-578, May 1990.

[30] D. Middleton, "Procedures for determining the parameters of the firstorder canonical models of class A and class B electromagnetic interference," IEEE Trans. Electromagn. Compat., vol. EMC-21, no. 3, pp. 190-208, Aug. 1979.

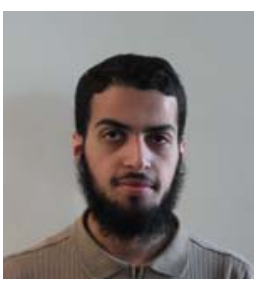

Suhail Al-Dharrab received the B.Sc. in Electrical Engineering from King Fahd University of Petroleum and Minerals, Dhahran, Saudi Arabia in 2005 and M.A.Sc. in Electrical and Computer Engineering from University of Waterloo, Waterloo, Canada, in 2008. He is currently a Ph.D. student in Electrical and Computer Engineering at University of Waterloo in Canada. His research interests span topics in cooperative communication, communication in impulsive noise, diversity techniques, spacetime coding, information theory and channel coding.

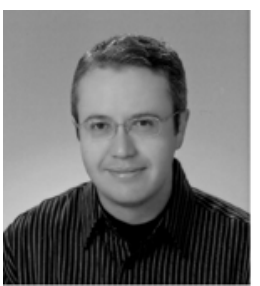

Murat Uysal was born in Istanbul, Turkey in 1973. $\mathrm{He}$ received the B.Sc. and the M.Sc. degree in electronics and communication engineering from Istanbul Technical University, Istanbul, Turkey, in 1995 and 1998, respectively, and the Ph.D. degree in electrical engineering from Texas A\&M University, College Station, Texas, in 2001. Since 2002, he has been with the Department of Electrical and Computer Engineering, University of Waterloo, Canada, where he is now an Associate Professor. His general research interests lie in communications theory and signal processing for communications with special emphasis on wireless applications. Specific research areas include MIMO techniques, space-time coding, diversity techniques and coding for fading channels, cooperative communications, and free-space optical communications.

Dr. Uysal is an Associate Editor for IEEE TRANSACTIONS ON WIRELESS COMMUNiCATIONS and IEEE COMMUNiCATIONS LETTERS. He was a Guest Co-Editor for WILEY JOURNAL ON WIRELESS COMMUNICATIONS And Mobile Computing's Special Issue on "MIMO Communications" published in 2004. He is currently serving as a Guest Co-Editor for IEEE Journal on SElected AREas in Communications' Special Issue on "Optical Wireless Communications" to be published in 2010. Over the years, he has served on the technical program committee of more than 50 international conferences in the communications area. He recently cochaired IEEE ICC'07 Communication Theory Symposium and CCECE'08 Communications and Networking Symposium. Dr. Uysal is a Senior IEEE member. 